\title{
Selection for boar fertility and semen quality - the way ahead
}

\author{
W.L. Flowers \\ Department of Animal Science, North Carolina State University, Raleigh, NC 27695-7621, USA
}

\begin{abstract}
Critical needs for the swine industry in terms of boar fertility evaluations are validation of semen quality estimates with in vivo reproductive data; estimation of the relative fertility of boars; and elimination of sub-fertile ejaculates. Single sire matings are the best way to validate semen quality estimates with reproductive performance. Sampling about $20 \%$ of the population provides an accurate estimation of the variability among boars and should be sufficient for this purpose. In vitro tests that measure univariate characteristics of ejaculates including motility and morphology appear to be just as accurate as those that measure multivariate traits such as in vitro fertilization in terms of predicting boar fertility. Reasons for this observation may be related to how properties of sperm cells are influenced by the sow reproductive tract. Several seminal plasma proteins show strong correlations with boar fertility and hold potential for being developed into tests that can rank the relative fertility of boars. Almost $90 \%$ of the variation in boar fertility was explained when the proportion of motile and acrosome-reacted spermatozoa was combined with relative amounts of $28 \mathrm{kDa}, \mathrm{p} / 6.0$ and $55 \mathrm{kDa}, \mathrm{p} / 4.5$ seminal plasma proteins. Consequently, combining different complementary tests improves estimations of boar fertility. Motility estimates routinely performed in most A.I. centres are a reasonable technique for identification and elimination of sub-fertile ejaculates. However, the accuracy with which they currently are conducted within the swine industry needs improvement.
\end{abstract}

\section{Introduction}

Estimation of fertility in boars presents some unique challenges for the swine industry. Unlike its beef and dairy counterparts, single-sire matings are rare within the commercial sector. This is because standard industry practices such as multiple matings; large numbers of sperm per insemination; high boar replacement rates; and the use of pooled semen hinder their use. Consequently, current approaches concentrate on quantification of physiological aspects of spermatozoa that are thought to be important for fertilization. Although progress is being made, identification of techniques for estimating boar and semen fertility via this strategy remains elusive. One of the main obstacles is that most semen tests evaluate the ability of spermatozoa to successfully complete only one of many steps in a complex process. Deficiencies in other areas can reduce fertilization even if the characteristic being measured is conducive for its occurrence. 
One way to address some of these challenges is to consider what types of information the swine industry needs to critically assess boar and semen fertility and then determine which fertility tests might best meet these needs. This premise is based on the assumption that there is not a single, in vitro test that can accurately predict boar fertility. However, perhaps a series of different tests administered at various times could. Hence, the objectives of this review are two-fold: first, to outline, perhaps speculatively, a set of requirements for the swine industry in terms of male fertility evaluations; and second, to examine our current understanding of relationships among selected tests for estimating semen quality and boar fertility. Special emphasis will be placed on how these techniques might be incorporated into strategies that are harmonious with the constraints of modern boar management systems. Hopefully, the end result will be a summary of relevant information that outlines what is possible now in terms of selecting for boar fertility and semen quality and what needs to be done subsequently to enhance the process.

\section{Boar and semen fertility assessment needs}

In order to be successful, strategies for fertility evaluations have to provide relevant information that can be obtained and used within the framework of normal management systems for boars. Consequently, a brief overview of the productive life of boars in commercial A.I. centres is shown (Fig. 1). Genetic evaluation of growth and physical characteristics including structural soundness typically are completed between 4 and 5 months of age. This coincides with the time when boars are moved into isolation facilities (Safranski 2008). The isolation period varies from 30 to 90 days based on the health status of the production system. The current trend within the industry is to isolate boars for 60 days or longer due to the problems associated with managing the P.R.R.S. virus (Amass \& Baysinger 2006). During isolation, boars are trained for collection. When the isolation period is over, they enter production and are collected 2 to 5 times per week depending on sow breeding targets, season, genetic line, and other farm specific criteria (Knox et al. 2008). Finally, culling typically occurs when they are between 18 and 24 months old in order to maximize genetic improvement (Robinson \& Buhr 2005).

An ideal situation for the swine industry would be to have an accurate estimate of relationships between the reproductive performance of boars and various semen quality tests when they enter production at 8 to 9 months of age or shortly thereafter. In order to accomplish this, quantitative data in the form of farrowing rates and litter sizes must be available at this time. This arguably is the most critical requirement. Without it, all subsequent assessments of boar and semen fertility are qualitative without a definitive reference point.

It is important to note that in vivo fertility data does not have to be obtained from every boar to establish these relationships as long as the sampling strategy accounts for the variability within the entire population. The 60 to 90 day isolation period provides an opportunity to obtain in vivo fertility data without influencing daily semen production destined for commercial farms. Semen collected from boars during this time typically is discarded so it would be available for "test" matings. In contrast, techniques that can accurately rank boars in terms of their relative fertility should be performed on several ejaculates from all boars during the isolation period with one occurring just prior to, or shortly after, their entry into the A.l. centre. Once in vivo fertility data become available from the subset of boars, the production of live pigs can be estimated for all boars based on their relative rank.

During the 10 to 16 month period that boars are producing semen destined for commercial farms, identification of sub-fertile ejaculates is important. This provides the main source of quality control and insures that ejaculates with low fertilizing potential are not used for inseminations. 


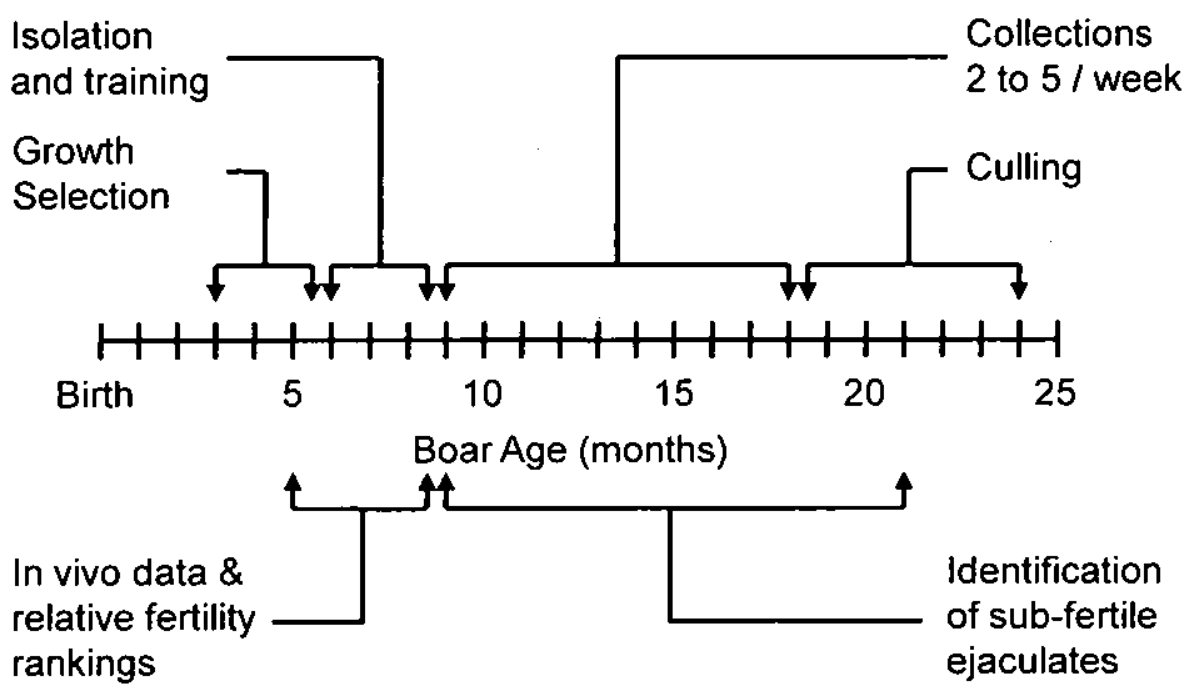

Fig. 1 Time line of productive life for modern boars housed in A.I. centres. Key management events are contained above the time line. Critical needs for evaluating boar fertility and semen quality are shown below the time line.

Screening for sub-fertility needs to be done on every ejaculate that is collected. These tests need to be performed accurately by A.I. centre personnel and their results have to be available immediately due to the short time interval between collection and production of insemination doses. It also may be necessary to reassess the relative fertility ranking of selected boars periodically during this phase. This would provide a way to monitor deviations from their fertility potential and make appropriate adjustments.

In summary, the three critical needs for boar fertility evaluations are to validate semen quality estimates with in vivo reproductive data; rank individuals in terms of their relative fertility; and identify and eliminate sub-fertile ejaculates. The first two should be met before or shortly after boars enter production, while the third one has to be addressed daily throughout their productive lives (Fig. 1). Consequently, determining which current techniques might be able to meet these critical needs is the next logical step in selecting for boar fertility and semen quality.

\section{Obtaining in vivo fertility data for validation of semen quality estimates}

Despite the reluctance of the swine industry to use of single-sire matings, the birth of live pigs is the best way to establish accurate reference points for male reproductive performance and validate in vitro fertility assessments. Results from a study conducted in a 200-head boar stud illustrate the importance of this process (Flowers 2002). In this particular study, each ejaculate with a motility score of $70 \%$ or greater was used to make insemination doses containing 1 to 9 billion total spermatozoa and used to breed between 75 and 100 sows from each insemination dose per boar. Changes in the number of pigs born alive in response to increasing the number of spermatozoa from 6 representative boars are shown (Fig. 2). The insemination dose at which litter size reached its peak and the actual number of pigs born alive at the optimal dose varied considerably among boars. These data demonstrate that there is a significant amount of variation in the fertility of boars currently used within the swine industry. Of particular interest was the identification of boars that demonstrated exceptional fertility at low insemination 


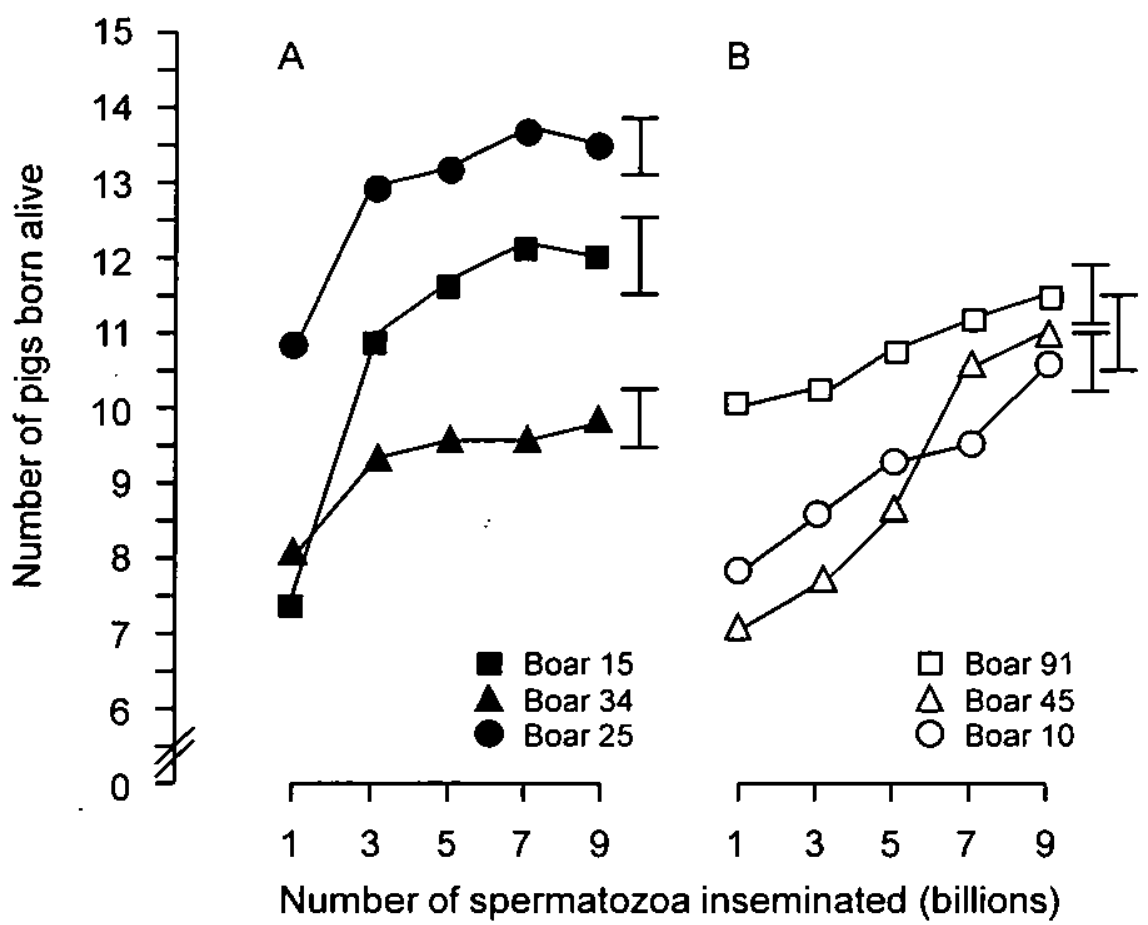

Fig. 2 Asymptotic (panel A) and linear (panel B) relationships between insemination doses and litter size (mean \pm S.E.M.) in selected boars. (with permission from Flowers 2008).

doses generally thought to be suboptimal and their counterparts with poor results with doses commonly accepted as industry standards.

Ideally, it would be nice to have these data for all boars in an A.I. centre before they enter production. However, this is not practically or economically feasible. An alternative approach would be to evaluate a subset of the boars in the population. Fig. 3 contains frequency distributions for the farrowing index of boars used in the previous study. The farrowing index is an estimate of the live pigs a boar produces each time his semen is used to breed sows and is calculated by multiplying the farrowing rate by the average number of pigs born alive.

The goal of any sampling strategy is to obtain a subset that is representative of the entire population. In the context of boar fertility, a practical approach would be to consider boars as being sub-fertile; fertile; or exceptionally fertile. By industry standards, a reasonable estimation of sub-fertile would be a farrowing index of 8.0 or lower. This is because the decision boundaries for farrowing rate and number of pigs born alive are generally accepted as being $80 \%$ and 10 pigs (PigCHAMP 2008). Similarly, a farrowing index of 11 seems logical for the exceptionally fertile category. It corresponds to a $92 \%$ farrowing rate and 12 pigs born alive which often are used as reproductive targets in the industry (PigCHAMP 2008). If these groupings are applied to the farrowing index data (Fig. 3), then a random sample of 40 boars, or $20 \%$, from this population should result in about 10,23, and 6 boars from the sub-fertile, fertile, and exceptionally fertile groups, respectively. This should be sufficient to obtain reliable in vivo fertility data from boars in each classification and thereby an estimate for the entire population.

The insemination dose used to obtain in vivo fertility estimates deserves careful thought. In the above example, a dose of 3 billion total spermatozoa was inseminated because it is considered the industry standard (Knox et al. 2008). However, use of insemination doses lower than 3 billion 


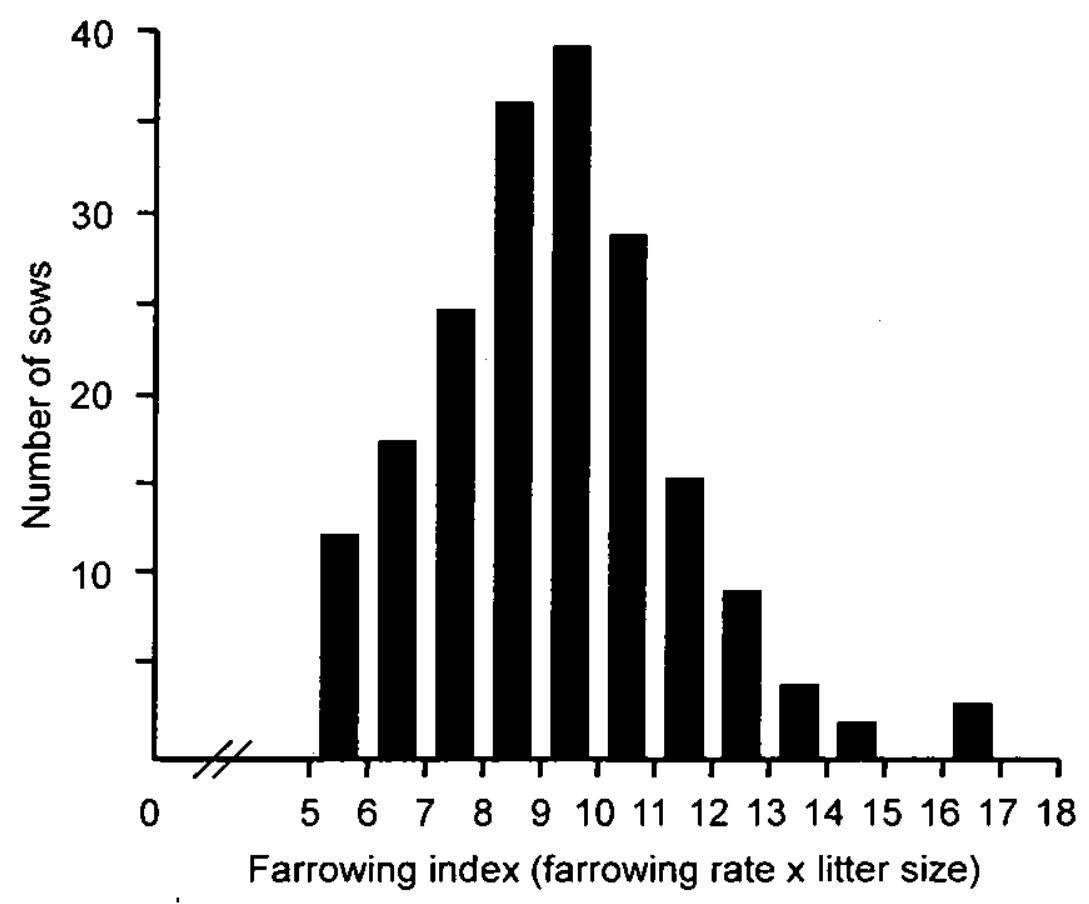

Fig. 3 Frequency distribution for farrowing index (farrowing rate $x$ litter size) from a population of modern A.I. boars $(n=200)$.

spermatozoa enhances the identification of fertility differences among boars and increases the predictive value of many in vitro semen assessment techniques (Xu et al. 1998, Flowers 2002, Popwell \& Flowers 2004, Ruiz-Shanchez et al. 2006). This decision, in part, depends on the relative importance of knowing the exact number of live pigs produced versus enhancing the ability of semen quality estimates to identify and rank fertile boars. Increasing the precision and accuracy of techniques for estimating boar fertility should also increase the number of live pigs produced.

\section{Semen quality estimates for ranking relative boar fertility}

A number of different techniques have been used to estimate the fertilizing ability of boar spermatozoa and, therefore, hold potential for identifying relative fertility differences. Excellent reviews describing the technical aspects associated with these tests and the physiology they mimic during fertilization are available (Rodriquez-Martinez 2003, Gadea 2005, Petrunkina et al. 2007, Foxcroft et al. 2008). What has not been addressed in as much detail is whether these tests can be integrated into a strategy to improve fertility estimates. Consequently, this possibility deserves further exploration.

\section{Fertility estimates based on properties of spermatozoa}

Selected techniques for estimating the fertility of boars by measuring various aspects of spermatozoa are summarized in Table 1. All are based on the premise that the proportion of sperm cells that possess a certain characteristic is either positively or negatively correlated with the ability of the ejaculate to fertilize ova. Those that measure motility, morphology, chromatin 
structure, and surface proteins rely on estimation of a single trait. Others such as sperm binding, ooctye penetration, and in vitro fertilization estimate functional aspects of spermatozoa which, in essence, are the combination of many individual traits. Consequently, it is logical to assume that the ones that measure functional aspects would be better suited for ranking boar fertility than those that measure a single trait.

Table 1. Selected techniques for estimating semen quality and their relationship with in vivo fertility.

\begin{tabular}{|c|c|c|}
\hline Technique & $\begin{array}{l}\text { Correlation with } \\
\text { In Vivo Fertility }\end{array}$ & Refierences \\
\hline Motility 7 to 10 days after storage & $r=0.36100 .46$ & $\begin{array}{l}\text { Xu et al. } 1998 \\
\text { Ruiz-Sanchez et al. } 2006\end{array}$ \\
\hline Normal morphology at collection & $r=0.59$ & Xu et al. 1998 \\
\hline Spermatozoa with cytoplasmic droplets at collection & $r=-0.25$ & Ruiz-Sanchez et al. 2006 \\
\hline Spermatozoa with normal DNA structure & $r=0.27$ to 0.91 & Evensen et al. 1994 \\
\hline Sperm plasma membrane proteins & $r=0.38$ to 0.53 & Ash et al. 1994 \\
\hline Ubiquitin bound to spermatozoa & $r=-0.31$ to -0.38 & Lovercamp et al. 2007 \\
\hline Oocyte membrane binding assay & $r=0.80$ & Berger et al. 1996 \\
\hline In vilro fertilization - male pronuclear formation & $r=0.35$ to 0.41 & Ruiz-Sanchez et al. 2006 \\
\hline
\end{tabular}

Surprisingly, the correlation coefficients between estimates of semen fertility and in vivo reproductive data do not support this assumption (Table 1). Those associated with a single physical aspect of spermatozoa were similar to those measuring their functional properties. Moreover, in the studies that used multiple regression techniques, motility and morphology estimates explained a large portion of the total variation observed in farrowing rates and number of pigs born alive in the population of boars being studied (Xu et al. 1998, Ruiz-Shanchez et al. 2006).

It is important to recognize that in vivo estimates of fertility were obtained via different methods for several of these studies. Work conducted by Xu et al. (1998) and Ruiz-Shanchez et al. (2006) used suboptimal insemination doses of 1.5 to 2 billion spermatozoa to obtain farrowing rates and litter sizes from their population of boars. In contrast, studies reporting very high correlations for normal DNA structure (Evensen et al. 1994) and oocyte binding (Berger et al. 1996) used heterospermic inseminations and paternity testing to estimate fertility in their boars. In vivo estimates of fertility varied from 2 to $98 \%$ of piglets sired with heterospermic inseminations, whereas farrowing rates and litter size normally were 70 to $90 \%$ and 9 to 12 pigs, respectively, with homospermic inseminations. Consequently, quantitative differences among correlations for some of these tests may be related to the manner in which in vivo estimates of boar fertility were obtained.

Nevertheless, the lack of consistent and significant advantages of multivariate techniques such as in vitro fertilization over those measuring univariate characteristics like motility has important implications for boar fertility evaluation. An underlying assumption for all semen tests is that characteristics of sperm cells measured in vitro reflect what happens to them in vivo. One fundamental difference between these two situations is the interaction of spermatozoa with the female reproductive tract, especially the oviduct. It is obvious that sperm-oviduct interactions are critical for successful fertilizations (Rodriquez-Martinez et al. 2005). What is not as clear is whether certain aspects of sperm function are influenced to a greater degree than others during their interaction with the female reproductive tract.

Results from a study originally designed to investigate the effect of the capacitation environment on in vitro fertilization efficiency may provide some insight into this question (Popwell 1999, Popwell \& Flowers 2001). Briefly, ejaculates from boars were split and either processed for in vitro fertilization or used to inseminate sows. At selected time intervals after insemination, spermatozoa were recovered from the oviducts of sows or removed from the in vitro fertilization system and evaluated. Progressive forward motility decreased in both environments over time and tended to be 
lower for spermatozoa recovered from the oviduct compared with those in the in vitro fertilization system (Fig. 4, top panel). in contrast, capacitation occurred very quickly in vitro, whereas a more protracted pattern was observed in vivo (Fig. 4, bottom panel).
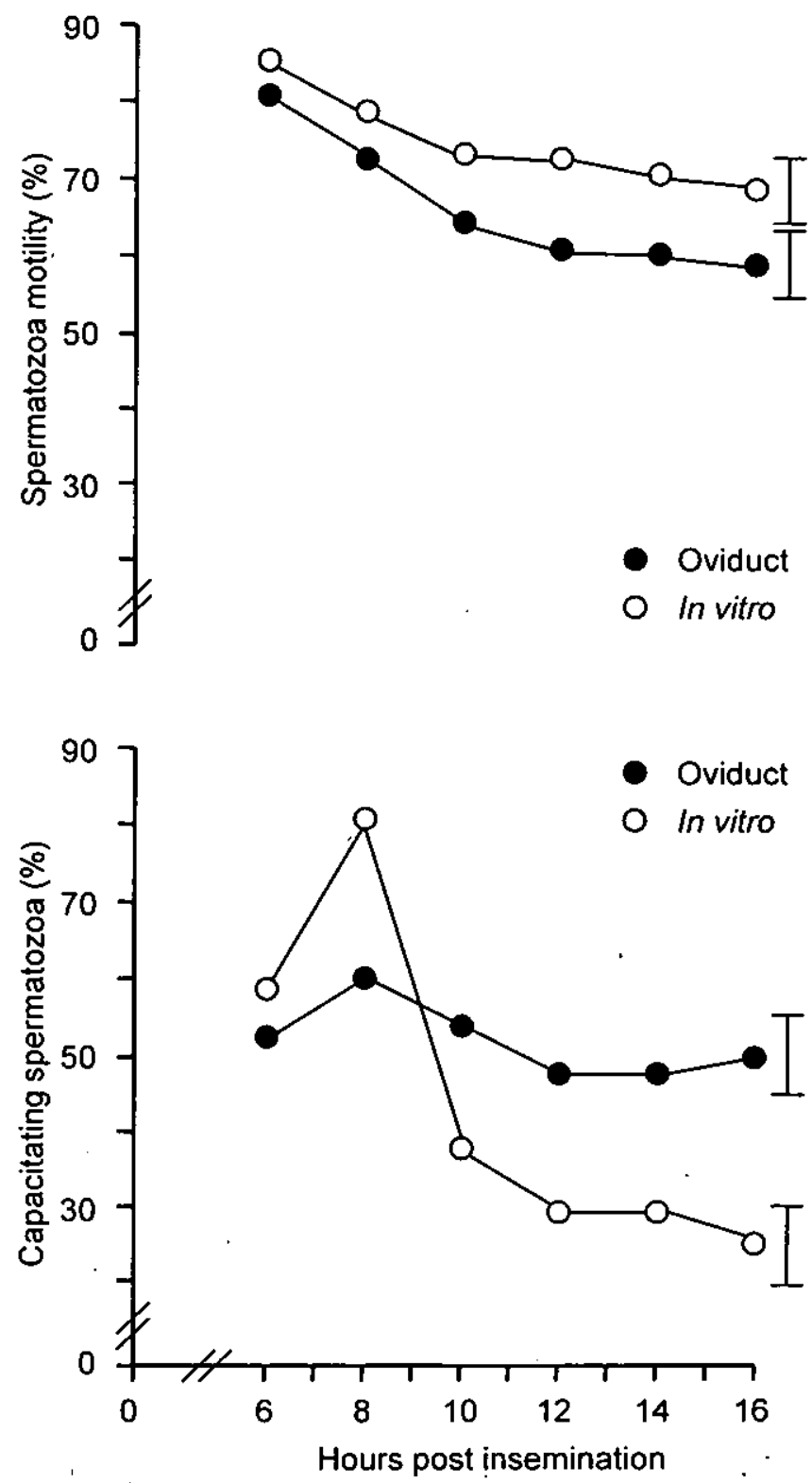

Fig. 4 Changes over time (mean \pm S.E.M.) in the proportion of spermatozoa exhibiting progressive forward motility (top panel) and undergoing capacitation (bottom panel) after incubation in vitro and recovery from the oviducts (adapted from Popwell 1999 and Popwell \& Flowers 2001).

These data help explain, in part, the lack of differences between univariate and multivariate tests in terms of their abilities to rank boar fertility. Estimates for progressive forward motility were equivalent between the in vitro and in vivo environments. Consequently, when the proportion of motile spermatozoa is estimated prior to insemination, this seems to be an 
accurate reflection of the population present in the oviduct prior to fertilization. In contrast, the time course over which capacitation occurs in vitro does not appear to be representative of what occurs in vivo. Thus, there is variability in how the oviduct influences different functions of spermatozoa. Consequently, a component missing from functional tests that select for boar fertility is an estimation of how spermatozoa interact with the oviduct after insemination. Development of cell culture methodologies has potential to address this deficiency and preliminary investigations indicate that variations in the binding of sperm cells to oviductal explants in vitro are associated with boar fertility differences (Waberski et al. 2005). However, whether binding in vitro accurately reflects all the changes spermatozoa undergo while in the oviduct remains to be determined.

\section{Fertility estimates based on seminal plasma proteins}

Proteins in seminal plasma have been shown to influence many important processes associated with fertilization including regulating uterine function after mating (Rozeboom et al. 1998, Woelders \& Matthijs 2001), ovulation (Waberski 1997), capacitation (Topfer-Petersen et al. 1998, Vadnais et al. 2005), oviductal binding (Petrunkina et al. 2001), and sperm-oocyte interactions (Caballero et al. 2004, Caballero et al., 2008). Moreover, when seminal plasma from boars of high fertility was used to replace that from boars of low fertility and vice versa, numbers of pigs born were increased and decreased, respectively (Flowers 1997). As a result, there has been increasing interest in using these as potential fertility markers for boars.

Selected seminal plasma proteins that have shown significant positive or negative correlations with farrowing rates and litter sizes are shown in Table 2. For the most part, correlation coefficients between relative amounts of these proteins and boar fertility are considerably stronger than those reported for various characteristics of spermatozoa (Table 1). This is particularly impressive because all the boars used in three of the studies had excellent motility and morphology estimates by industry standards (Flowers 1995, Ruiz-Sanchez 2006 as cited by Foxcroft et al. 2008, Turner \& Flowers 2009).

Table 2. Relationships between relative amounts of selected seminal plasma proteins and in vivo fertility.

\begin{tabular}{|c|c|c|}
\hline Seminal Plasma Proteins & $\begin{array}{l}\text { Correlation with } \\
\text { In Vivo Fertility }\end{array}$ & References \\
\hline 20 kDA, pl 6.0 & $r=-0.76$ & Foxcroft et al. 2008 \\
\hline 25-29 kDA, pl 5.9-6.2 & $r=0.45$ to 0.60 & $\begin{array}{l}\text { Flowers } 1995 \\
\text { Foxcroft et al, } 2008 \\
\text { Turner \& Flowers } 2009\end{array}$ \\
\hline 55 kDA, pl 4.5-5.1 & $r=0.56$ to 0.62 & $\begin{array}{l}\text { Flowers } 1995 \\
\text { Turner \& Flowers } 2009\end{array}$ \\
\hline 60 kDA, pl 5.9 & $r=-0.66$ & Foxcroft et al. 2008 \\
\hline
\end{tabular}

It is clear that seminal plasma proteins modulate several important aspects of how spermatozoa interact with the oviduct after insemination. Consequently, it is tempting to speculate that variations in these proteins may reflect differences in the abilities that spermatozoa have to accomplish these tasks and, thus, prepare themselves for fertilization. Clearly, additional studies are required to validate this speculation. However, if it is correct, then quantification of specific seminal plasma proteins may provide a way to estimate how the oviduct and other parts of the female reproductive tract interact with spermatozoa after insemination, information that is clearly lacking from techniques currently used for male fertility evaluations. 
Fertility estimates based on properties of spermatozoa and seminal plasma proteins

It is clear that techniques discussed previously have strengths and weaknesses in terms of their ability to rank the relative fertility of boars. In terms of those that estimate the properties of spermatozoa, motility and normal morphology appear to be just as good as those that measure more complicated aspects of sperm functions possibly because the latter do not account very well for the contributions of the oviduct. In contrast, some seminal plasma proteins show strong correlations with boar fertility and may be reflective of how effectively spermatozoa can undergo changes necessary for fertilization once they enter the sow. Thus, using combinations of these two types of assays should, in theory, enhance the ability to rank the relative fertility of boars.

A recent study investigated this possibility using heterospermic inseminations and subsequent paternity testing with 12 boars (Turner \& Flowers 2009). Seminal plasma proteins and selected attributes of spermatozoa were measured in every ejaculate used for breeding. Relative concentrations of 26-28 kDa, p/6.0 $\left(r^{2}=0.66, p<0.001\right)$ and 55-57 kDa, p/ $5.6\left(r^{2}=0.05, p\right.$ $<0.05)$ proteins; the proportion of spermatozoa exhibiting an acrosome reaction $\left(r^{2}=0.15, p\right.$ $<0.001)$; and the proportion of motile spermatozoa $\left(r^{2}=0.03, p<0.075\right)$ explained $89 \%$ of the variation in the relative fertility in this population of boars. As mentioned previously, use of heterospermic inseminations and paternity testing has the advantage of increasing the variability in fertility compared to using farrowing rates and litter sizes. As a result, caution needs to be used when attempting to extrapolate these results to commercial situations using homospermic inseminations. However, the potential of combining several complementary techniques to rank the relative fertility of boars deserves a careful evaluation by the swine industry.

\section{Semen quality estimates for identifying sub-fertile ejaculates}

Acute and chronic stresses can temporarily reduce the fertility of boars (Flowers 1997). As a result, there is a need for procedures that can accurately screen for sub-fertile ejaculates. In theory any of the techniques discussed for ranking the relative fertility of boars could be used to accomplish this. However, the average time between collection and when the decision to keep or discard an ejaculate needs to be made is less than 1 hour in most commercial A.I. centres (Knox et al. 2008). This time limitation effectively eliminates all of the assays discussed previously (Tables 1 and 2) with the exception of motility and morphology evaluations.

Estimates of motility, albeit in various forms, are common in A.I. centres, while morphological evaluations are performed much less frequently and often in situations where a problem that isn't reflected by motility evaluations is expected (Knox et al. 2008). Therefore, it appears that the swine industry has procedures in place to provide data for meeting this critical need. Thus, the relevant question becomes how accurate are these routine evaluations for motility.

Results from a field study provide data to address this question (Flowers 1994). Video footage of ejaculates with different motilities were used either in training sessions or sent to personnel responsible for evaluating semen quality in A.I. centres in the southeastern U.S. Participants were asked to provide quantitative (actual percentages) and qualitative (keep or reject) estimates of motilities for each video segment. None of the participants were using CASA systems in their studs at the time the study began. The largest variances were associated with samples whose actual motilities were between 60 and $70 \%$ (Table 3). This was also the range in which the most mistakes were made in terms of keeping or rejecting ejaculates. Finally, there was a general tendency for technicians to overestimate motility. 
Table 3. Accuracy and precision of boar sperm motility estimates by A.I. centre technicians in the southeastern U.S. (adapted from Flowers 1994).

\begin{tabular}{cccc}
\hline $\begin{array}{c}\text { Percentage of } \\
\begin{array}{c}\text { Motile Spermatozoa } \\
\text { in the Ejaculate }\end{array}\end{array}$ & $\begin{array}{c}\text { Motility Estimate by } \\
\text { Technician } \\
(\%, \text { mean }+ \text { SEM) }\end{array}$ & $\begin{array}{c}\text { Proportion of Technicians } \\
\text { keeping Ejaculate }\end{array}$ & $\begin{array}{c}\text { Proportion of Technicians } \\
\text { rejecting Ejaculate }\end{array}$ \\
\hline 80 & $90+8$ & $150 / 150$ & $0 / 150$ \\
70 & $83+15$ & $98 / 150$ & $52 / 150$ \\
60 & $75+20$ & $84 / 150$ & $66 / 150$ \\
50 & $62+9$ & $29 / 150$ & $121 / 150$ \\
40 & $32+7$ & $0 / 150$ & $150 / 150$ \\
\hline
\end{tabular}

Previous studies have demonstrated that when insemination doses of 3 billion spermatozoa or higher are used, relationships between motility and reproductive performance in sows is asymptotic (Flowers 1997, Xu et al. 1998). The point at which fertility no longer increases at appreciable rates with increasing motilities is between 60 and $70 \%$. Consequently, it appears that the accurate assessment of motility by A.I. centre personnel under field conditions is challenging, especially when ejaculates are close to physiologically relevant levels related to fertility. This compromises the ability of the swine industry, at least in the southeastern U.S., to meet the critical need of identifying sub-fertile ejaculates. Technologies associated with CASA have improved, while their costs have decreased over the past 10 years. These systems offer potential for improving the ability of A.I. centres to accurately estimate motilities and minimize the use of sub-fertile ejaculates.

\section{Conclusion}

An ideal situation for the swine industry would be able to have an accurate estimation of boar fertility by the time they enter A.I. centres around 9 months of age combined with the daily identification of sub-fertile ejaculates thereafter. Of these, the industry has the technology to identify sub-fertile ejaculates on a daily basis via routine assessment of motility. However, the accuracy at which this is currently being done within the industry is questionable and could be improved.

Development of tests that accurately rank the relative fertility of boars and procedures to validate these data with reproductive performance are necessary. The isolation period which typically lasts 60 to 90 days provides a reasonable time frame over which these data can be obtained without compromising semen used for breeding commercial sows. Single sire matings from about $20 \%$ of the boars being evaluated should be sufficient to obtain a good estimate of the variability in reproductive performance within the population. These data are viewed as critical in that they provide definitive reference points for all subsequent in vitro estimates of boar and semen fertility.

Ranking the relative fertility of all boars destined to enter production also needs to be done during the 60 to 90 day isolation period. This can be achieved with in vitro evaluations. Unfortunately, at the present time, there does not appear to be a single test that provides accurate estimates for relative fertility including those that measure the ability to fertilize ova in vitro. The proportion of motile spermatozoa after 7 to 10 days of storage appears to be as good as any other single test in terms of its predictive value. Moreover, it can be performed by most A.I. centres without additional investments in equipment or technical expertise. Most of the other techniques that can provide a relative ranking of boar fertility would likely have to be outsourced to external laboratories because they require specialized equipment and highly trained technicians. 
Use of seminal plasma proteins as boar fertility markers is still a theoretical concept. However, it does show promise. It is an attractive technology from an industry perspective because if proteins with high correlations with male fertility are identified, then it should be possible to develop elisa-based tests that could be performed quickly and easily at A.I. centres. When seminal plasma protein data were combined with other estimates of semen quality the accuracy of predicting boar and semen fertility was improved significantly. Consequently, the way ahead for selecting for boar and semen fertility is through the application of multiple techniques performed at selected times during the productive life of boars.

\section{References}

Amass SF \& Baysinger A 2006 Swine disease transmission and prevention. In Diseases of Swine, Ninth Edition, pp 1075-1098 Eds Straw BE, Zimmerman JJ, D'Allaire S and Taylor DJ. Oxford: Blackwell Publishing Ltd.

Ash KL, Berger T, Horner CM \& Famula TR 1994 Boar sperm plasma membrane profile: correlation with the zona-free hamster ova assay. Theriogenology 42 1217-1226.

Berger T, Anderson DL \& Penedo MCT 1996 Porcine fertilizing potential in relationship to sperm functional capabilities. Animal Reproduction Science $\mathbf{4 4}$ 231-239.

Caballero I, Vazquez IM, Gil MA, Calvette II, Roca I \& Sanz L 2004 Does seminal plasma PSP-I/ PSP-II spermadhesin modulate the ability of boar spermatozoa to penetrate homologous oocytes in vitro? Journal of Andrology 25 1004-1012.

Caballero I, Vazquez IM, Garcia EM, Parilla I, Roca J, Calvete JI, Sanz L \& Martinez EA 2008 Major proteins of seminal boar plasma as a tool for biotechnological preservation of spermatozoa. Theriogenology 70 1352-1355.

Evenson DP, Thompson L \& Jost L 1994 Flow cytometric evaluation of boar semen by the sperm chromatin structure assay as related to cryopreservation and fertility. Theriogenology 41 637-651.

Flowers WL 1994 What technologies are available today to evaluate boar fertility? Proceedings, 1994 American Association of Swine Veterinarians Annual Conference 2-17.

Flowers WL 1995 Relationships between seminal plasma proteins and boar fertility. In Department of Animal Science Annual Swine Report pp 22-27 Ed See, MT. Raleigh:North Carolina State University.

Flowers WL 1997 Management of boars for efficient production. Journal of Reproduction and Fertility Supplement 52 67-78.

Flowers WL 2002 Increasing fertilization rate in boars: influence of number and quality of spermatozoa inseminated. lournal of Animal Science 80 (E Supplement 1) 47-53.

Flowers WL 2008 Genetic and phenotypic variation in reproductive traits of $\mathrm{Al}$ boars. Theriogenology 70 1297-1303.

Foxcroft GR, Dyck MK, Ruiz-Sanchez A, Novak S \& Dixon WT 2008 Identifying useable semen. Theriogenology
$701324-1336$.

Gadea J 2005 Sperm factors related to in vitro and in vivo porcine fertility. Theriogenology 63 431-444.

Knox R, Levis D, Safranski T \& Singleton W 2008 An update on North American boar stud practices. Theriogenology 70 1202-1208.

Lovercamp KW, Safranski T), Fischer KA, Manandhar G, Sutovsky M, Herring W \& Sutovsky P 2007 Arachidonate 15-Jipoxygenase and ubiquitin as fertility markers in boars. Theriogenology 67 704-718.

Petrunkina AM, Gehlhaar R, Drommer W, Waberski D \& Töpfer-Petersen E 2001 Selective sperm binding to pig oviductal epithelium in vitro. Reproduction 121 889-896.

Petrunkina AM, Waberski D, Gunzel-Apel AR \& TöpferPeterson E 2007 Determinants of sperm quality and fertility in domestic species. Reproduction 134 3-17.

PigCHAMP 2008 Benchmark: setting higher standards for pork production, 2008 edition. http://www.pigchamp. $\mathrm{com} /$ docs/Benchmark_2008_edition.pdf.

Popwell JM 1999 Porcine semén quality estimates and capacitation environments and their relationship to fertilization in vitro. Doctoral Dissertation, North Carolina State University, Raleigh, NC.

Popwell JM \& Flowers WL 2001 Effect of capacitation environment of spermatozoa on fertilization of porcine oocyte in vitro. Journal of Animal Science 79 (Supplement 1) 229.

Popwell JM \& Flowers WL 2004 Variability in relationships between semen quality and estimates of in vivo and in vitro fertility in boars. Animal Reproduction Science $8197-113$.

Robinson JAB and Buhr MM 2005 Impact of genetic selection on management of boar replacement. Theriogenology 63 668-678.

Rodriguez-Martinez H 2003 Laboratory semen assessment and prediction of fertility: still utopia? Reproduction in Domestic Animals 38 312-318.

Rodriguez-Martinez H, Saravia F, Wallgren M, Tienthai P, Johannisson A \& Vazquez IM 2005 Boar spermatozoa in the oviduct. Theriogenology 63 514-535.

Rozeboom KJ, Troedsson MH \& Crabo BG 1998 Characterization of uterine leukocyte infiltration in gilts after artificial insemination lournal of Reproduction and Fertility $\mathbf{1 1 4}$ 195-199.

Ruiz-Sanchez AL 2006 Semen assessment techniques 
for determining relative boar fertility. Doctoral Thesis Edmonton, Alberta: University of Alberta.

Ruiz-Sanchez AL, O'Donoghue R, Novak S, Dyck MK, Cosgrove JR \& Dixon WT 2006 The predictive value of routine semen evaluations and IVF technology for determining relative boar fertility. Theriogenology $66736-748$.

Safranski T 2008 Cenetic selection of boars. Theriogenology $701310-1316$.

Topfer-Petersen E, Romero A, Varela PF, EkhlasiHundrieser M, Dostalova Z, Sanz L \& Calvette II 1998 Spermadhesions: a new protein family. Facts, hypotheses and perspectives. Andrologia 30 217-224.

Turner FB \& Flowers WL 2009 Relationships among common semen quality estimates, seminal plasma proteins and boar fertility. Animal Reproduction Science (submitted).
Vadnais ML, Kirkwood RN, Templeman RJ, Specher D) \& Chou K 2005 Effect of cooling and seminal plasma on the capacitation status of fresh boar sperm as determined by chlortetracycline staining. Animal Reproduction Science 87 121-132.

Waberski D 1997 Effects of semen components on ovulation and fertilization. Journal of Reproduction and Fertility Supplement 52 105-109.

Waberski D, Magnus F, Ardon F, Petrunkina AM, Weitze KF \& Topfer-Petersen E 2005 Importance of sperm-binding assays for fertility prognosis of porcine spermatozoa. Theriogenology 63 470-484.

Woelders H \& Matthijs A 2001 Phagocytosis of boar spermatozoa in vitro and in vivo. Reproduction Supplement 58 113-127.

Xu X, Pommier S, Arbov T, Hutchings B, Sotto W \& foxcroft GR 1998 In vitro maturation and fertilization techniques for assessment of semen quality and boar fertility. Journal of Animal Science 76 3079-3089. 